\title{
Comparisons of VRP Optimization Algorithmic Methods for the Optimal Routing of Multiple Delivery Vehicles with Time Constraint
}

\author{
Nuttachat Wisittipanit*, Adirek Baisukhan, Chanin Srisuwannapa \\ School of Science, Mae Fah Luang University, Chiang Rai, Thailand \\ (Received 30 September 2020, accepted 28 December 2020) \\ https://doi.org/10.36224/ijes.130401
}

\begin{abstract}
Transportation costs account for a large portion of business expense in any logistics firm; thus, achieving proper solutions that manage those transportation activities well and reduce such expense should be the number one priority for the business. Essentially, such logistics management involves the routing plans for company vehicles that perform delivery/pick up and also the number of vehicles utilized. This study investigated and compared the optimization performances of routing algorithms using simulated geographic data based in Chiang Rai, Thailand, emulating the post office operation which had 1 post office, 4 delivery vehicles and 2 delivery zones ( 2 vehicles per zone): 65 customer locations for zone A and 74 for zone B. The major objective of this particular routing problem, called Vehicle Routing Problem (VRP), was that the total delivery distance of those 4 delivery vehicles combined should be minimized; moreover, those vehicles mush finish their delivery operation within a time constraint, set at 2 hours. The optimization algorithms, employed for the routing procedures, were Large Neighborhood Search (LNS), Particle Swarm Optimization (PSO), Differential Evolution (DE) and Ant Colony Optimization (ACO), combined into 3 hybrid algorithms (LNS|PSO, LNS|DE and LNS|ACO). Those hybrid algorithms including pure ones (DE, PSO and ACO) were compared for their optimization performances; and the results showed that LNS|ACO hybrid algorithm was superior than the other two hybrid ones and also far better than pure DE, PSO and ACO algorithms at various parameter variants. Additionally, most algorithms (hybrid and pure ones) finished the delivery routing within the time constraint of 2 hours except only for the pure algorithms at minimum parameter variant.
\end{abstract}

Keywords: Large Neighborhood Search, Differential Evolution, Particle Swarm Optimization, Ant Colony Optimization, Vehicle Routing Problem, Optimization, Logistics

\section{Introduction}

Transportation market constitutes approximately 5,000 billion US dollars in the industry size [1]. And the main business cost of the logistics industry is apparently the transportation expenses, comprising fuel and labor as well as vehicle depreciation costs. For big logistics companies, they have to control movements of a large group of vehicles; and such vehicle management heavily involves route planning and necessary number of vehicles required to deliver/pick up at target locations. In essence, they need decent procedures to manage the daunting operation of vehicle routing in order to improve their financial well-being. This study concentrated on obtaining the optimized solutions for Vehicle Routing Problem (VRP) involving a post office operation using simulated geographic data based in Chiang Rai, Thailand; such simulated data included 1 post office, 4 delivery vehicles and 2 zones of delivery ( 2 vehicles per zone). There were4optimization algorithms involved in this study: Large Neighborhood Search (LNS), Differential Evolution (DE) and Particle Swarm Optimization (PSO) and Ant Colony Optimization (ACO), in which some of them were formed to be hybrid algorithms (LNS|DE, LNS|PSO, LNS|ACO). The results demonstrated that the routing optimization performances of 
LNS|ACO hybrid algorithm were better than those of hybrid algorithm counterparts and far better than those of DE, PSO and ACO "pure" algorithms at distinct parameter variants which were the number of vector populations and iterations as well as LNS parameters including number of destroyed nodes and LNS iterations. Note that the optimization performance refers to the objective value being the total delivery distance of all 4 vehicles; and the optimized objective value or the least total delivery distance was the result of the LNS|ACO hybrid with maximum parameter variants. Moreover, the delivery time constraint of 2 hours could be achieved by most algorithms except only for the pure algorithms (DE, PSO and ACO) at minimum parameter variant.

\section{Literature review}

In logistics-related business, transportation expenditure is generally a significant part of the total operation costs; thus, the lessening of such cost can not only considerably increase the financial health of logistics firms, but also help reduce the environment impact due to lessen carbon emission [2]. To solve such issue, optimization methods for Vehicle Routing Problem or VRP [3] might be a practical solution. VRP refers to the means of searching for optimum routing solutions, given a group of vehicles that travel to destinations in order to deliver or pick up packages. The objective or optimal routing solution is usually the least total travelled distance; however, other objectives include time durations and financial gains. In term of computational complexity, finding the optimal routing resolution to the VRP is considered to be NP-hard (Nondeterministic Polynomial acceptable problem) [4] which means that using only the mathematical optimization to solve is limited to certain problem sizes which are generally small. However, the problem sizes of real-world applications are commonly large; for instances, the depots of a large electronics supply company consisting of hundreds of delivery vehicles with thousands of items as cargos, or a big pharmaceutical firm having thousands of vehicles to deliver vaccines to multiple hospitals. Therefore, solving of the VRP by only exact mathematics may not be practical; and as such, meta-heuristic algorithms are considered to be more practical for the optimization procedure.

\subsection{Large Neighborhood Search}

Large Neighborhood Search or LNS algorithm [5] is an optimization algorithm that might be the most suitable for the VRP since its algorithmic process suits large problem sizes and mostly deals with nodes and edges (where nodes can represent locations and edges represent travel routes). In the optimization procedure for VRP, LNS reiteratively changes an existing route solution into a novel one (called a neighborhood solution) using various transformation strategies. Usually, a transformed routing solution is relatively close to the original one; however, its objective value can be either better or worse than the previous solution. But with the large amount of transforming iterations, the routing solution could eventually get better; even though most of the solutions obtained from LNS might not be the exact optimum solution but many studies considered them to be adequately efficient [6]. The main disadvantage point of LNS is that some transformations might be conducted at improper places and become too large and so the optimization process is at high risk of moving farther and farther away from the global optimum [7]. Some of the transformation methods for LNS include destroy/repair and swap strategies. 


\subsection{Particle Swarm Optimization}

Particle Swarm Optimization or PSO algorithm [8] is an optimization algorithm based on a random searching method in a population of candidate solutions (called particles) that resembles the individuals' movements in a swarm of animals such as birds or fish. Each particle in a swarm represents only one solution and it possesses 2 features: velocity (as a vector) and position, in which its movement is affected by the cognitive information of its past experience and also the swarm perception in order to achieve a better position. For the algorithmic process of PSO, the velocities and positions of all particles are iteratively updated and those particles would move according to their new calculated velocities until the stopping criteria are met.

\subsection{Differential Evolution}

Differential Evolution or DE algorithm [9] is an evolution-based optimization algorithm that imitates the evolutionary process of organisms which includes the components of mutation, cross-over and selection. The procedure of evolution begins with a randomly initiated population (or a group of solutions) of defined size $(N)$ and each solution is a $D$-dimensional vectors where each of its item is a real number in $[0,1]$ range; in other words, those items in the vectors are randomly generated to fall in that range in the beginning of the process. Then, the population of solutions iteratively evolves into a new group of population where a better solution, if exists, is selected from that group. Note that there are various methods to mutate a vector into a new one; one method is to combine a randomly chosen vectors ( 2 or 3 vectors) using a scaling function.

\subsection{Ant Colony Optimization}

Ant Colony Optimization [10] is an optimization algorithm that mimics the behavior of a colony of ants when traversing to find food source from its nest in which they place pheromones along the path to guide other ants. When ants leave pheromone on the trails, over time it would evaporate; however, as time passes by, the shorter routes (to the food) get used more often resulting in the higher density of pheromone than that of the longer routes. Thus, the route would converge to the optimal one (shortest or close to the shortest) given enough time. It is one of the probabilistic models for determining solutions of complex numerical problems. The algorithm works by creating artificial ants or simulation agents that search for optimized solutions; and during the solution searching, those artificial ants "lay down pheromone" or document their positions such that in later computational iterations, other ants could find better solutions. Their fascinating behavior is similar to that of the honey bee which is also a social insect.

\section{Materials and methods}

This study emphasized on the solving of VRP problems concerning the simulated data having a post office and 2 delivery zones: zone A with 65 addresses and zone B with 74 addresses which included both residential and commercial addresses. Additionally, there were 4 delivery vehicles. All the address data were strategically picked from the actual geo-coordinates based in Chiang Rai city, Thailand, in order to partially imitate the real operation of the Chiang Rai post office. All the chosen locations are displayed on the Google Earth Pro®application [11] as shown in Figure 1 where the yellow pin specifies the post office of Chiang Rai city; the red and green pins specify the customer locations belonging to zone A and zone B, respectively. Moreover, each zone was assumed to possess 2 delivery vehicles, responsible for distributing submitted packages 
from the post office to customer locations; additionally, those vehicles must deliver packages within a time constraint which was set to be 2 hours (120 minutes) from 10am to $12 \mathrm{pm}$. Therefore, the major objective of this particular VRP problem was that the total travel distance of those 4 delivery vehicles once all the packages were distributed was at the minimum and such delivery operation must be completed within a set time frame.

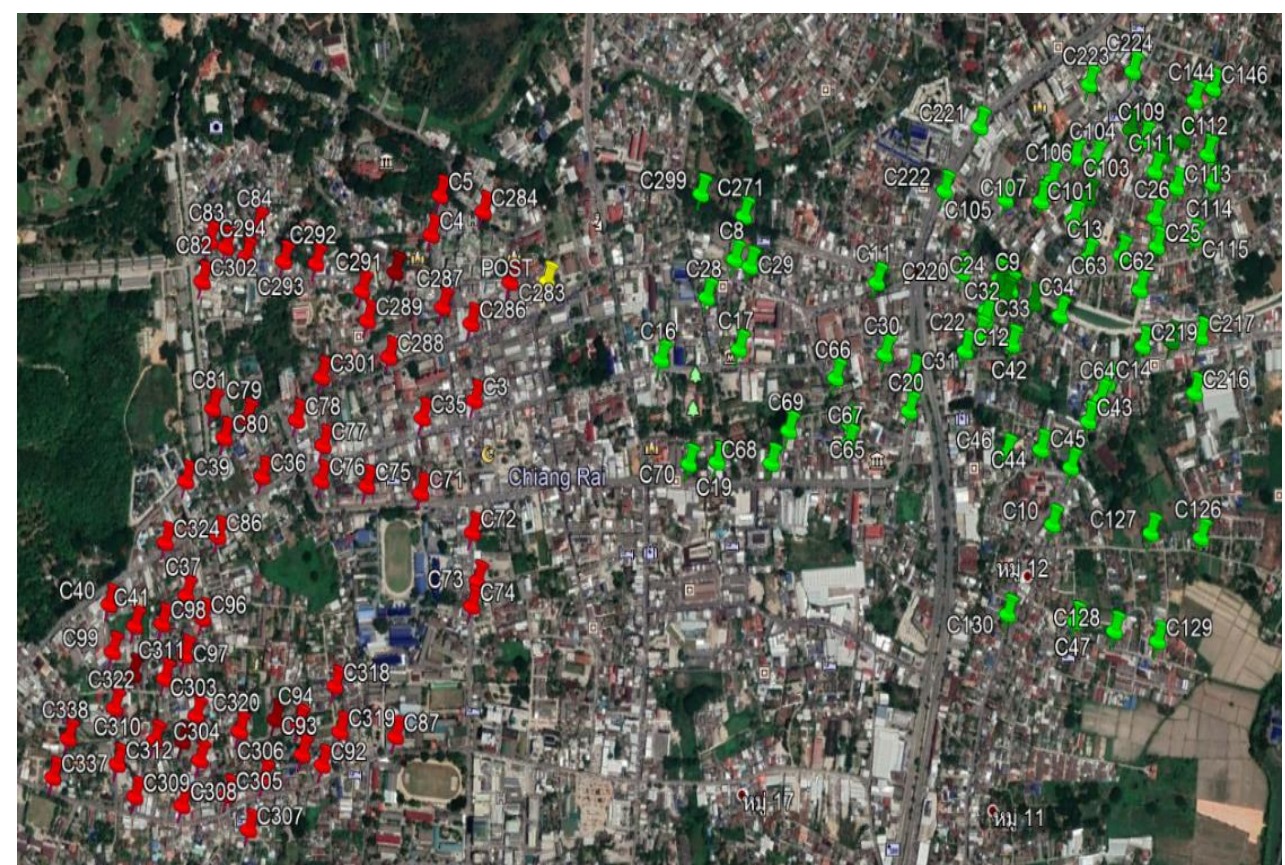

Figure 1: Simulated geo-locations in Chiang Rai province, Thailand: post office (yellow pin), 65 customer addresses (red pins) for zone A and 74 customer addresses (green pins) for zone B, all displayed in Google Earth Pro® software.

According to Figure 1, all pin locations, including that of the post office, link to geographic coordinates attained from Google Earth Pro ${ }^{\circledR}$ application. For zone A, there are 66 coordinates ( 65 addresses +1 post office address); and for zone B, there are 75 coordinates (74 addresses +1 post office address). Then, 2 distance matrices were constructed, one for each zone; the first one has $(65+1) \times(65+1)$ dimensions and the second one has $(75+1) \times(75+1)$ dimensions. For each matrix, a cell value of $\mathrm{i}^{\text {th }}$ row and $\mathrm{j}^{\text {th }}$ column signifies a distance between $\mathrm{i}^{\text {th }}$ location and $j^{\text {th }}$ location, respectively. Figure 2 shows an example of a matrix having $(4+1) \times(4$ +1 ) dimensions (1 post office, 3 customer locations).

\begin{tabular}{|c|c|c|c|c|}
\hline & Post & C1 & C2 & C3 \\
\hline Post & - & 4.2 & 2.5 & 3.2 \\
\hline C1 & 4.2 & - & 2.8 & 3.7 \\
\hline C2 & 2.5 & 3.1 & - & 1.8 \\
\hline C3 & 3.2 & 3.7 & 1.9 & - \\
\hline
\end{tabular}

Figure 2: An example of a distance matrix (in $\mathrm{km}$ ) of 5 x 5 dimensions with 1 post office and 3 customer locations

According to Figure 2, each element of the matrix is the shortest distance between a pair of geographic coordinates; for instances, $4.2 \mathrm{~km}$ (row 2, column 3) is the shortest distance from 
the post office to customer location 1 and also from customer location 1 to the post office (row 3, column 2). All shortest distances in the matrix was calculated by the Google Maps API [12] with the Google maps library using a Python programming script. However, the distance matrix is not symmetric because the route from address A to address B might not be similar to that from address $\mathrm{B}$ to address A; for example, the one-way traffic could be enforced in some roads. This matrix plays an essential role in the VRP optimization procedure by being the main data of shortest distances between all addresses such that the main objective, achieving minimum total distances of all the 4 delivery vehicles.

\section{Numerical experiments and results}

This study was focused on solving VRP of the simulated post office operation consisting of 4 vehicles and 2 delivery zones: one with 65 customer addresses and the other with 74 customer addresses; andall the addresses were real geo-locations in Chiang Rai city, Thailand. The main objective for the VRP optimization was to minimize the total delivery distances for all those 4 vehicles within a given time frame i.e. 3 hours. This studyemployed 3 meta-heuristic algorithms: (1) DE (2) PSO and (3) ACO, and 3 hybrid ones: (1) LNS|DE (2) LNS|PSO and (3) LNS|ACO, to solve such particular VRP. Additionally, the performances of all those algorithms were compared.

For DE, PSO and ACO algorithms, 2 major parameters: popsize and maxiter played essential roles in their optimization performances; and for the hybrid algorithms, there were additional 2 parameters for the LNS algorithm part: maxiterLNS and lnsdestroy. The parameter bigRepgoverns how many hybrid operations to be run. All of the optimization proceduresand algorithms were written in Python programming language [13].

\subsection{Application of LNS|DE hybrid to VRP}

The LNS|DE hybrid routing optimization procedure, shown in Figure 3, begins with the DE algorithm where a number of vectors, equaling topopsize $=10,50,200$ witheach vector having the size of $2+65=67$ for zone A (the number of delivery vehicles + number of customer locations) and $2+74=76$ for zone $\mathrm{B}$, are initialized randomly (each item of a vector with the value ranging from 0 to 1). Next, integer indexes starting from 0 are assigned to an individual item in each section depending on the item's value (from low to high). Then, each customer section is split into 2 sub-sections for 2 delivery vehicles ( 2 delivery vehicles per one zone); and those 2 sub-sections are assigned to vehicles according to the vehicles' index order. Once all the assignments are done, each vector is considered to be a routing solution ready to be evaluated for the objective value - the total delivery distance. Each vehicle would travel, starting from the post office, to customer locations according to their assigned indexes in each vector. Then, DE looks for the global best solution which is essentially a vector corresponding to the routing solution having the least total delivery distance. Next, each vector goes through the process of mutation, crossover and selection such obtain trial vectors which are evaluated again for their objective values. If a trial vector produces the objective value better than that obtained by the current vector, the current vector would become that trial vector; DE then again updates the global best routing solution. This optimization process keeps iterating until the number of iterations is equal to the maxiterparameter (set to $10,50,200$ ). 


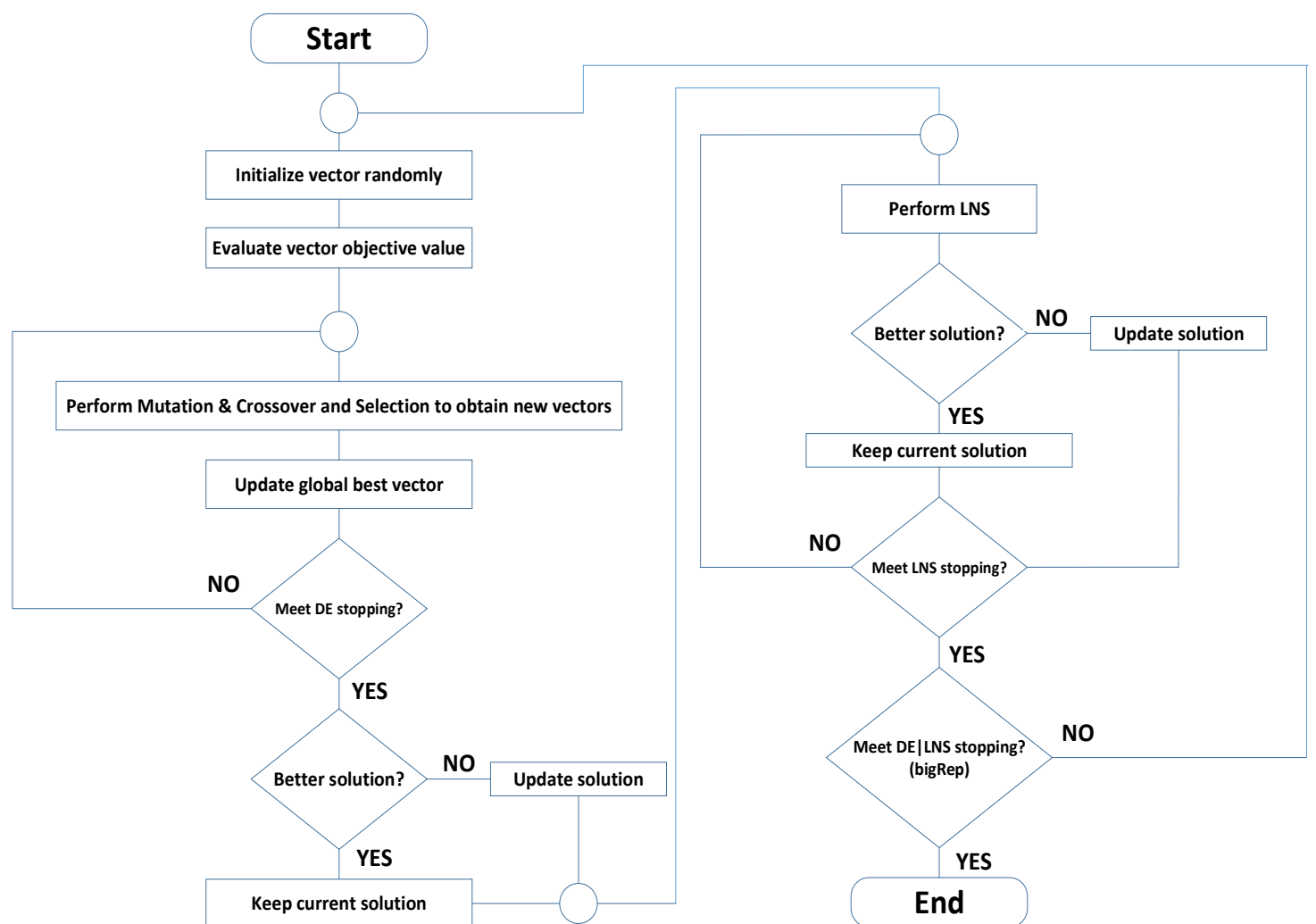

Figure 3: The flowchart of routing optimization for LNS|DE hybrid

The global best solution is then sent to LNS algorithm where only one transformation method is applied: DESTROY/REPAIR; in the process, random nodes are partially destroyed according to the Insdestroy parameter (set to 50\%). Then LNS repairs the routing by inserting the destroyed nodes one by one; the insertion method is done in a way that the total delivery distance of the repaired route is at minimum. The DESTROY/REPAIR process keeps iterating until the iteration amount met maxiterLNS (set to 50,100,200). Once the LNS procedure is done, the entire LNS|DE hybrid optimization process would evaluate if the number of the hybrid iterations meets the bigRep parameter or not; if it is equal, then, the entire process is finished. Note that for the pure DE process, the routing optimization steps end at "Meet DE stopping?" block.

\subsection{Application of LNS|PSO hybrid to VRP}

The LNS|PSO hybrid routing optimization process, as shown in Figure 4, starts with the initialization of particles to have random positions with zero velocity; the number of particles (or vectors) is set to be equal to popsize $=10,50,200$. The size and assignment of each vector is similar to those done in LNS|DE process. Then, the particles are evaluated for their objective values - total delivery distance in order for the LNS|PSO to determine the global best solution. The process would check if the stopping condition is met (number of iterations equals to maxiterparameter, set to $10,50,200$ ); if still not met, the procedure performs the update of guidance and velocity/position of all the particles and evaluates them again. The global best solution from PSO is then transferred to the LNS process which is the same one as conducted in the LNS|DE routing optimization steps. 


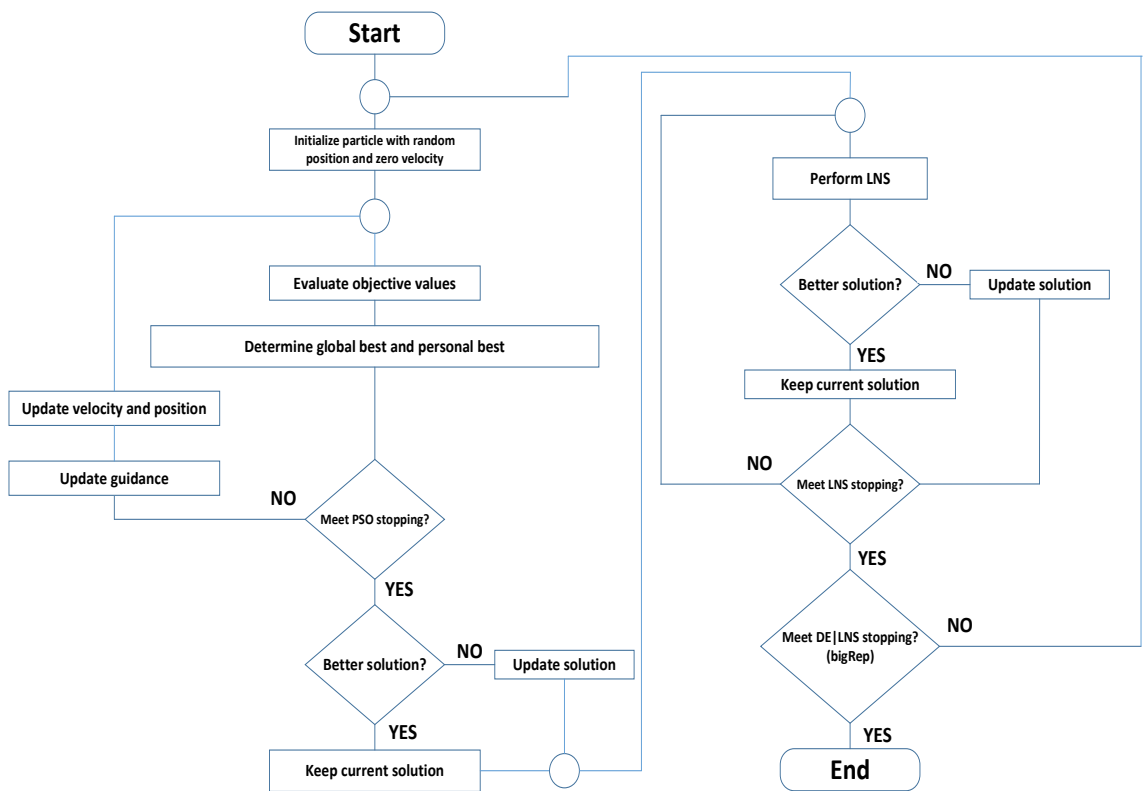

Figure 4: The flowchart of routing optimization for LNS|PSO hybrid.

\subsection{Application of LNS|ACO hybrid to the defined VRP}

The LNS|ACO hybrid VRP optimization flowchart, as shown in Figure 5, starts with the initialization of number of ants (vectors), set to be equal to popsize $=10,50,200$, in which each ant stochastically generate a solution. The size and element assignments of each vector is similar to those done in LNS|DE process. Then, the ants are evaluated for their objective values where they get compared in order for the LNS|ACO to determine the global best solution. The process would stop if the stopping condition is met which refers to the number of iterations being equal to maxiterparameter, set to $10,50,200$; if the condition is still not met, the pheromone update gets updated and those ant agents would construct solutions again. The global best solution from ACO is then sent to the LNS procedure which is similar to that conducted in the LNS|DE routing optimization steps.

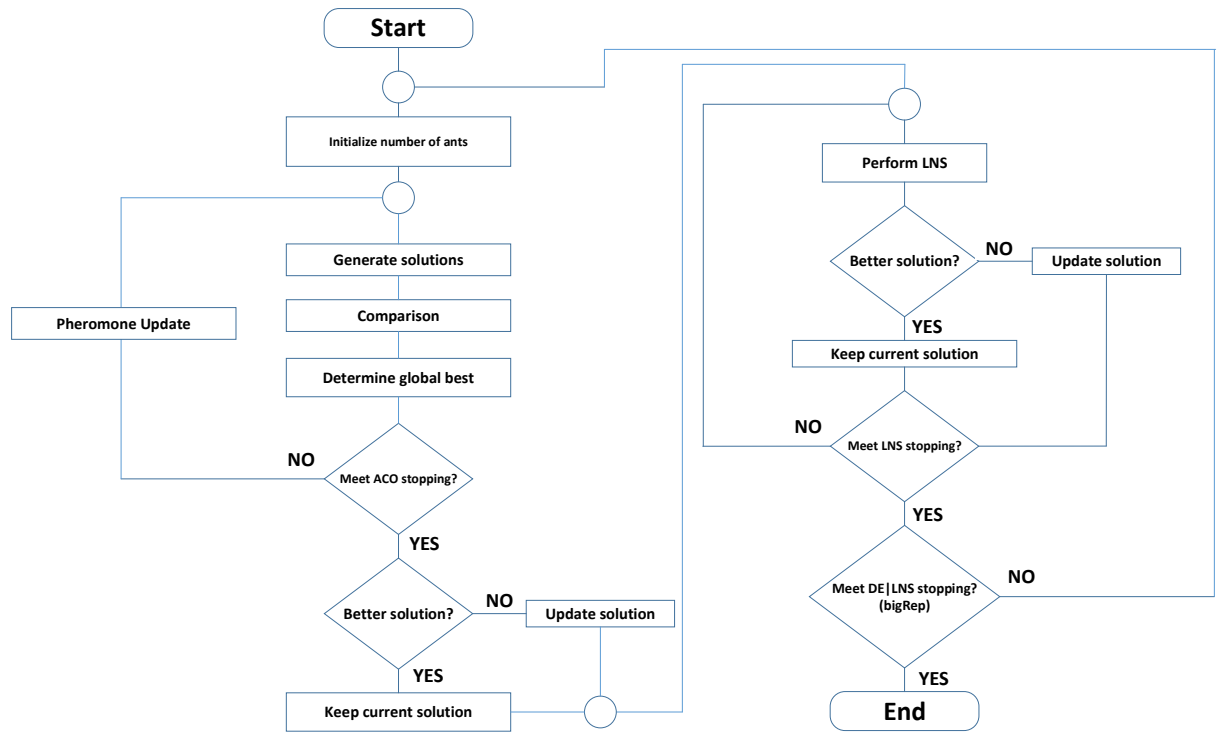

Figure 5: The flowchart of routing optimization for LNS|PSO hybrid 


\subsection{Results}

The numerical experiment of the routing optimization employed the LNS|DE hybrid, LNS|PSO hybrid, LNS|ACO hybrid, DE, PSO and ACO algorithms at distinct parameter variants:popsize $=10 \mid$ maxiter $=10$, popsize $=50 \mid$ maxiter $=50$, andpopsize $=200 \mid$ maxiter $=$ 200(note that for only the hybrid algorithms, maxiterLNS, Insdestroy and bigRepparameters are set to $50,50 \%$ and 5 , respectively). The results in Table 1 show that the performances of routing optimization using the hybrid algorithms (LNS|DE, LNS|PSO, LNS|ACO) for all pure parameter variants(popsize and maxiter) are far better than those of pure DE, PSO and ACO; and most the performances of both LNS|ACO hybrid and pure ACO algorithms are slightly better than those of the hybrid and pure algorithm counterparts (except at popsize $=10$ and maxiter $=10$ ). The best optimization performance belongs to LNS|ACO hybrid, when maxiterLNS $=50$, which is at $31.84 \mathrm{~km}$ withpopsize $=200$ and maxiter $=200$. This is expected since more vector populations and iterations should lead to superior objective values. For the maxiterLNSvariants at 100, 200 and 400 (popsize $=200$, maxiter $=200$, lnsdestroy $=50 \%$ and bigRep $=5$ ), the performances of LNS|ACO hybrid are slightly better than those ofLNS|DE and LNS|PSO hybrids with the best objective value being $31.40 \mathrm{~km}$ at maxiterLNS $=400$. The LNS|DE and LNS|PSO hybrids demonstrate a peculiar result at maxiterLNS $=200$ having the better performance than that atmaxiter $L N S=100$, in which the reason might be that at some point in the LNS optimization procedure, the solutions could moves farther away from the global optimum point.

Table 1: Results of the routing optimization for 2 zones (zone A and B) using LNS|DE, LNS|PSO,LNS|ACO,DE, PSO and ACO with various parameter variants

\begin{tabular}{|c|c|c|c|c|c|c|}
\hline \multirow{3}{*}{$\begin{array}{c}\text { Parameter } \\
\text { variants }\end{array}$} & \multicolumn{6}{|c|}{ Global best solution (total delivery distance in kilometers) } \\
\hline & popsize & maxiter & popsize & maxiter & popsize & maxiter \\
\hline & 10 & 10 & 50 & 50 & 200 & 200 \\
\hline LNS|DE & \multicolumn{2}{|c|}{36.40} & \multicolumn{2}{|c|}{34.12} & \multicolumn{2}{|c|}{32.25} \\
\hline LNS|PSO & \multicolumn{2}{|c|}{37.54} & \multicolumn{2}{|c|}{34.05} & \multicolumn{2}{|c|}{33.64} \\
\hline LNS|ACO & \multicolumn{2}{|c|}{36.68} & \multicolumn{2}{|c|}{33.98} & \multicolumn{2}{|c|}{31.84} \\
\hline DE & \multicolumn{2}{|c|}{92.16} & \multicolumn{2}{|c|}{75.45} & \multicolumn{2}{|c|}{68.41} \\
\hline PSO & \multicolumn{2}{|c|}{89.23} & \multicolumn{2}{|c|}{77.12} & \multicolumn{2}{|c|}{70.24} \\
\hline \multirow[t]{2}{*}{$\mathrm{ACO}$} & \multicolumn{2}{|c|}{90.84} & \multicolumn{2}{|c|}{74.95} & \multicolumn{2}{|c|}{67.49} \\
\hline & \multicolumn{6}{|c|}{ Global best solution (popsize $=200$, maxiter $=200$ ) } \\
\hline \multirow{2}{*}{$\begin{array}{c}\text { maxiterLNS } \\
\text { variants }\end{array}$} & \multicolumn{2}{|c|}{ maxiterLNS } & \multicolumn{2}{|c|}{ maxiterLNS } & \multicolumn{2}{|c|}{ maxiterLNS } \\
\hline & \multicolumn{2}{|c|}{100} & \multicolumn{2}{|c|}{200} & \multicolumn{2}{|c|}{400} \\
\hline LNS|DE & \multicolumn{2}{|c|}{32.18} & \multicolumn{2}{|c|}{32.85} & \multicolumn{2}{|c|}{32.04} \\
\hline LNS|PSO & \multicolumn{2}{|c|}{33.50} & \multicolumn{2}{|c|}{33.70} & \multicolumn{2}{|c|}{32.98} \\
\hline LNS|ACO & \multicolumn{2}{|c|}{31.89} & \multicolumn{2}{|c|}{31.80} & \multicolumn{2}{|c|}{31.40} \\
\hline
\end{tabular}

As this particular VRP also has time constraint, set to be 2 hours from 10 am to 12 $\operatorname{pm}(120$ minutes) in which all the vehicles must finish their parcel delivery, the results for travelling time demonstrated in Table 2,show that all but 3 pure algorithms: DE, PSO and ACO, exceeds time constraint of 120 minutes at 129.4, 126.47 and 128.08, respectively. Note that to 
calculate the travelling time, the duration of picking the parcel and deliver to customer by the vehicle drivers was taken into account; and it was set to be approximately 30 seconds.

Table 2: Results of the travelling time of 4 vehicles for 2 zones (zone A and B, 2 vehicles for each zone) using LNS|DE, LNS|PSO, LNS|ACO, DE, PSO and ACO with various parameter variants

\begin{tabular}{|c|c|c|c|c|c|c|}
\hline \multirow{3}{*}{$\begin{array}{c}\text { Parameter } \\
\text { variants }\end{array}$} & \multicolumn{6}{|c|}{ Travelling time (minutes) } \\
\hline & popsize & maxiter & popsize & maxiter & popsize & maxiter \\
\hline & 10 & 10 & 50 & 50 & 200 & 200 \\
\hline LNS|DE & \multicolumn{2}{|c|}{73.64} & \multicolumn{2}{|c|}{71.36} & \multicolumn{2}{|c|}{69.49} \\
\hline LNS|PSO & \multicolumn{2}{|c|}{74.78} & \multicolumn{2}{|c|}{71.29} & \multicolumn{2}{|c|}{70.88} \\
\hline LNS|ACO & \multicolumn{2}{|c|}{73.92} & \multicolumn{2}{|c|}{71.22} & \multicolumn{2}{|c|}{69.08} \\
\hline $\mathrm{DE}$ & \multicolumn{2}{|c|}{129.4} & \multicolumn{2}{|c|}{112.69} & \multicolumn{2}{|c|}{105.65} \\
\hline PSO & \multicolumn{2}{|c|}{126.47} & \multicolumn{2}{|c|}{114.36} & \multicolumn{2}{|c|}{107.48} \\
\hline \multirow[t]{2}{*}{$\mathrm{ACO}$} & \multicolumn{2}{|c|}{128.08} & \multicolumn{2}{|c|}{112.19} & \multicolumn{2}{|c|}{104.73} \\
\hline & \multicolumn{6}{|c|}{ Travelling time $($ popsize $=200$, maxiter $=200)$} \\
\hline \multirow{2}{*}{$\begin{array}{c}\text { maxiterLNS } \\
\text { variants }\end{array}$} & \multicolumn{2}{|c|}{ maxiterLNS } & \multicolumn{2}{|c|}{ maxiterLNS } & \multicolumn{2}{|c|}{ maxiterLNS } \\
\hline & \multicolumn{2}{|c|}{50} & \multicolumn{2}{|c|}{100} & \multicolumn{2}{|c|}{200} \\
\hline LNS|DE & \multicolumn{2}{|c|}{69.42} & \multicolumn{2}{|c|}{70.09} & \multicolumn{2}{|c|}{69.28} \\
\hline LNS|PSO & \multicolumn{2}{|c|}{70.74} & \multicolumn{2}{|c|}{70.94} & \multicolumn{2}{|c|}{70.22} \\
\hline LNS|ACO & \multicolumn{2}{|c|}{69.13} & \multicolumn{2}{|c|}{69.04} & \multicolumn{2}{|c|}{68.64} \\
\hline
\end{tabular}

\section{Discussion and conclusion}

This study was focused on the experiments involving the routing optimization of 4 delivery vehicles travelling to multiple customer addresses in 2 zones from the post office, using the simulated geographic data based on real locations in Chiang Rai, Thailand. The simulated data include1 post office (Chiang Rai city location), 4 delivery vehicles ( 2 per zone), 65 customer locations for zone A and 74 customer locations for zone B. The optimization procedure utilized 3 pure algorithms: DE, PSO and ACO, and 3 hybrid ones: LNS|DE, LNS|PSO and LNS|ACO; then, their performances were compared using distinct parameter variants. The LNS|ACO hybrid algorithms lightly outperformed the other 2 hybrid algorithms (LNS|PSO and LNS|ACO) in the same parameter variant and was obviously superior to the pure algorithms (DE, PSO and ACO). In addition, most algorithms had their vehicles finished the delivery process within the time constraint of 2 hours except for the pure algorithms at minimum parameter variant. That LNS|ACO hybrid algorithm had the best objective value was not surprising since ACO was more suitable to the VRP than the others. However, the actual optimized objective value i.e. total travelling distance might not be achieved; therefore, a commercial solver capable of this particular problem size is required to achieve the exact optimal total distance value in order that its performance could be compared with those of metaheuristic algorithms used in this study.

\section{References}

[1] Logistics Market: Global Industry Trends, O., 2020. Logistics Market Size, Share | Industry Trends, Growth Report \& Forecast 2020-2025. [online] Imarcgroup.com. Available at: www.imarcgroup.com/logistics-market [Accessed 25 September 2020].

[2] Thakuriah P., Liao Y., Transportation Expenditures and Ability to Pay: Evidence from Consumer Expenditure Survey, Transportation Research Board, 1985(1): 257-265, 2006. 
[3] Dantzig G. B., Ramser J. H. The Truck Dispatching Problem, Management Science, 6 (1): 80-91, 1959.

[4] Bovet D. P., Crescenzi P. Introduction to the Theory of Complexity. Prentice Hall. p. 69. ISBN 0-13-915380-2, 1994.

[5] Ahuja R., Ergun O., Orlin J., Punnen A., A survey of very largescale neighborhood search techniques, Discrete Appl. Math., 123, 75-102,2002.

[6] Boden P., Hahne H., Rank S., Schmidt T., Dispatching of Multiple Load Automated Guided Vehicles Based on Adaptive Large Neighborhood Search, Operations Research Proceedings 2019, Springer Cham,https://doi.org/10.1007/978-3-030-48439-2_45, 2019.

[7] Lippenberger M. P., An Adaptive Large Neighborhood Search for Routing and Scheduling Carsharing Service Requests, Operations Research Proceedings 2018, Springer Cham,https://doi.org/10.1007/978-3-030-185008_2, 2018.

[8] Kennedy J., Eberhart R., Particle SwarmOptimization, Proceedings of the IEEE International Conferenceon Neural Networks, Piscataway, 1995.

[9] Storn R., Price K., Differential Evolution - A Simple and Efficient Heuristic for global Optimization over Continuous Spaces, Journal of Global Optimization, 11, 341-359, 1995.

[10] Dorigo R., Maniezzo V., Colorni A., Ant System: Optimization by a Colony of Cooperating Agents, IEEE Transactions on Evolutionary Computation, 1(1): 53-66, 1997.

[11] Google Earth. 2020. Earth Versions - Google Earth. [online] Available at: www.google.com/earth/versions [Accessed 15July 2020].

[12] Google Developers. 2020. Google Developers. [online] Available at: developers.google.com [Accessed 15July 2020].

[13] Rossum G. V., Python tutorial: Technical Report CS-R9526, Centrumvoor Wiskunde en Informatica (CWI). Amsterdam, 1995. 